PARTIE II

$$
\begin{aligned}
& \text { Les récits, } \\
& \text { entre stratégie } \\
& \text { et action }
\end{aligned}
$$




\title{
Les Conditions
} de cohérence des récits stratégiques De la narration à la nar-action

\author{
"I have done the deed." \\ Shakespeare, Macbeth
}

\begin{abstract}
Cet article présente et examine les différents types de conditions de cohérence (internes compte tenu de la composition du récit, externes de type I qui traitent de la relation du récit à ses audiences, externes de type II qui sont liées à l'articulation du récit par rapport au réel et à l'action) dont le respect confère au récit sa capacité stratégique, transformant ainsi la narration en une nar-action, une narration par et pour I'action.
\end{abstract}

$\mathrm{L}$ es stratégies mises en œuvre ces dernières années par des firmes aussi diverses que Vivendi, HP, Renault ou Apple ont connu des fortunes variées, inversant ou confirmant les analyses initiales des observateurs (médias, analystes financiers, concurrents, partenaires, fournisseurs, clients). Dans les deux premiers cas, Vivendi et HP, les dirigeants dont les propositions étaient encensées au départ ont ensuite été désavoués par leur conseil d'administration. Dans les deux autres cas, Renault et Apple, les stratégies telles qu'elles étaient énoncées ont subi de fortes critiques à l'origine avant que les dirigeants qui les portaient ne rencontrent le succès et accèdent ainsi à un statut de quasi gourou. Comment expliquer ces remises en cause ou confirmation des perceptions initiales quant à ces stratégies et aux dirigeants qui les incarnent? À ce sujet, N. Giroux dans son texte argumente que «si les narrations sont "mémorables" car plus vivantes et concrètes que des chiffres, cela ne veut pas dire pour autant qu'elles soient véridiques » (Giroux et Marroquin, 2006, p. 30). Elle considère « qu'il serait éclairant de mettre en perspective la 
narration comme acte de langage, en la situant dans son articulation avec les autres activités des acteurs en présence, comme les actes politiques ou les actes d'allocation des ressources » (p. 32). Autrement dit, N. Giroux nous interroge sur le lien de la narration à l'action. Face à cette question, ce texte traite des conditions de cohérence internes et externes par lesquelles un récit devient stratégique et performatif. Il considère la narration comme une façon de structurer puis de communiquer une série de faits (Adam, 1984) et conçoit la stratégie comme une capacité à orienter l'action afin de transformer le réel. Il met en particulier l'accent sur les couplages entre narrateurs et audiences, entre fiction et réalité, entre narration et action comme conditions de la performance de la narration pour la stratégie. Il conduit à proposer le concept de naraction (narration par et pour l'action) pour le récit stratégique.

Dans une première partie sont présentées les conditions de cohérence interne d'une narration. Dans une seconde partie nous traitons des conditions d'un premier type de cohérence externe (cohérence externe de type 1) par lesquelles une narration rencontre ses audiences, parvient à les intéresser et à les intégrer en tant que parties prenantes pour son développement ultérieur. Dans une troisième partie, nous exposons les conditions d'un second type de cohérence externe (cohérence externe de type 2 ) par lesquelles, au-delà des audiences envisagées précédemment, s'organise le couplage de l'organisation à son environnement. L'entrée et la pénétration d'Apple dans le domaine de la distribution de musique en ligne est mobilisée comme trame pour illustrer notre propos. La stratégie d'Apple est basée sur la construction et la généralisation d'un format propriétaire apte à devenir un standard de fait. Sa diffusion repose sur la mise à disposition d'un site de distribution de musique en ligne offrant une alternative crédible au piratage, couplée au développement et à la production d'objets iconiques (iPod et iShuffle) conçus pour tirer le meilleur parti de ce site. Par cette stratégie, Apple se positionne face aux grands acteurs de l'électronique audio (Sony avec son Walkman, Philips avec sa $\mathrm{K} 7$ et son $\mathrm{CD}$ ) comme il l'était hier face à IBM dans l'informatique' ${ }^{1}$.

\section{I. - LES CONDITIONS DE COHÉRENCE INTERNE D'UN RÉCIT STRATÉGIQUE}

Dans un premier temps, nous relions l'apport de la narration à la stratégie à une triple capacité : 1) de sélection et de mise en relation de personnages et d'événements dans un décor ${ }^{2}, 2$ ) de construction et de mise en œuvre de sens au service d'une intention, 3) de légitimation du narrateur par rapport à son récit. Cet ensemble constitue selon nous pour le narrateur un premier jeu de conditions de cohérence nécessaires pour assurer la dimension et capacité stratégique du récit.

\section{Choix et mise en relation d'éléments au sein d'un récit}

Les éléments de la construction au sein du récit ainsi que les modalités de la cohérence

1. Une intrigue possible, à savoir la possibilité pour un challenger de détrôner Apple par un standard ouvert comme ce fut le cas avec Microsoft pour les systèmes d'exploitation n'est pas traitée ici.

2. La mise en intrigue qui structure le récit littéraire nous semble constituer un élément facultatif dans le récit stratégique. Elle n'est donc pas considérée dans ce texte. 
de celui-ci ont été au centre des travaux menés par les formalistes russes (Propp, 1970), puis par les structuralistes notamment en France. Ceux-ci s'intéressent aux éléments présents ainsi qu'aux mises en relation au sein du récit. Ils montrent que ceuxci suivent des formes récurrentes que ce soit pour les contes, pour les mythes ou pour l'approche littéraire de manière plus large.

Une organisation qui s'engage ou souhaite s'engager dans une trajectoire stratégique fait face à une double difficulté. D'un côté, une partie des éléments constitutifs du passé et du présent est pléthorique et ambigu. De l'autre, une partie des éléments constitutifs du futur, en particulier ceux qui seront le résultat de l'action stratégique ou de celle d'autres acteurs, n'existe pas encore. L'information à disposition est donc structurellement pléthorique, ambiguë et incomplète. Il faut donc à la fois choisir à partir de ce qui existe et d'autre part imaginer à ce qui pourrait être. Comme l'auteur d'un roman, l'une des tâches du stratège est donc de sélectionner et d'ordonner les différents éléments qu'il souhaite mobiliser, rencontrer ou envisage de rencontrer pour les intégrer progressivement en un même ensemble cohérent. Il faut planter un décor et convier ou accepter des personnages destinés à y vivre des événements dans une période. C'est la dimension démiurge du stratège comme du romancier. À partir de ces éléments, quatre types de mise en relation sont selon nous présentes au sein des narrations stratégiques.

Le premier type de mise en relation est temporel et chronologique, présentant les événements passés et à venir qui rythmeront la narration proposée. Qu'est ce que Apple va faire en premier qu'est ce qui adviendra en second? Qu'à été l'histoire d'Apple jusquelà et que va-t-il maintenant se passer? À quel rythme l'introduction des différents modèles d'iPod va-t-elle s'effectuer et l'ouverture des sites iTunes à travers le monde se produire? Comment s'insère l'introduction du iShuffle dans l'histoire plus large de l'iPod? Une narration, tout comme un calendrier, inscrit les personnages-acteurs et les événements au sein de séquences, permet le choix d'horizons plus ou moins lointains et de rythmes plus ou moins intenses sur une trajectoire, offre une capacité d'inscription des périodes de temps courts par rapport à celles des temps longs. Le second type de mise en relation est spatial, établissant les proximités entre les personnages-acteurs ainsi que les liens qui les unissent. Quels sont les lieux où se situent les centres de $R \& D$, de production, de distribution d'Apple? Comment s'insèrent-ils dans leur environnement? Comment et en quoi sont-ils reliés les uns aux autres? Une narration, tout comme une carte géographique, inscrit les personnages-acteurs et leurs liens dans l'espace et permet de multiplier les perspectives en insistant selon les cas sur les topographies, les voies de communication, les densités.

Le troisième type de mise en relation est celui des ressources et de leurs objets, établissant ce dont disposent les acteurs-personnages et ce à quoi ils le destinent. Quelles sont les ressources et compétences dont disposent les différents centres d'Apple? À destination de quels types de produits? Comment se fait l'intégration software/hardware selon les produits? Une narration, tout comme un tableau chiffré, permet d'attribuer des ressources, de caractériser leur intensité et de désigner leurs destinations.

Le quatrième type de mise en relation est causal, présentant la manière dont l'intervention des personnages-acteurs construit 
les événements et en retour la façon dont les événements commandent l'intervention des acteurs. L'existence de mini-disque dur permet de stocker l'information musicale en grande quantité dans une machine de taille réduite. Le développement des mémoires flash conduit Apple à proposer une déclinaison de l'iPod en iShuffle, plus petit, moins cher mais avec une capacité de stockage plus faible. Une narration tout comme un schéma, composé de ronds, de triangles et de flèches, permet de mettre en évidence les conséquences possibles A ou B si X se déplace en Y.

Dans tous ces registres, la narration agit comme un mode de représentation sous forme textuelle ou de discours. Les éléments (personnages, événements, décor, période) qui composent la narration pourraient tout autant se représenter selon les cas sous une forme visuelle dans un calendrier (dimension temporelle), dans une carte (dimension spatiale), dans un tableur (dimension des ressources et de leurs objets) ou encore dans un schéma (dimension causale). La narration se distingue de ces différents types de représentations en ce qu'elle permet d'intégrer en un seul ensemble les différents registres. Elle leur est par contre similaire en ce qu'elle permet la simulation en jouant sur la modification fictive, soit de l'un des éléments constitutifs, soit de l'un des modes de mise en relation. Si dans une narration alternative, Apple imagine d'annuler ou de reculer la sortie de l'un de ses nouveaux produits (iShuffle) de quelques mois, en quoi et comment cela va-t-il se répercuter sur la production et les ventes de ses propres produits (iPod) qu'il peut cannibaliser et sur celles des concurrents?

Le choix des éléments (personnages, événements, décor, période) et leur ordonnancement grâce aux différents types de mise en relation possibles construit la narration (figure 1). La structure du récit garantit la maîtrise de la diversité des éléments : assurant la cohésion, elle permet la mémorisation comme la lisibilité des énoncés (Adam, 1984). Ceci constitue ce que Ricœur dénomme première mimesis de la narration (Ricœur, 1983).

Pourtant, bien souvent, pour le narrateurstratège comme pour le romancier, les éléments et les mises en relation résistent ou s'échappent. L'histoire proposée avance, parfois tranquillement, parfois de manière

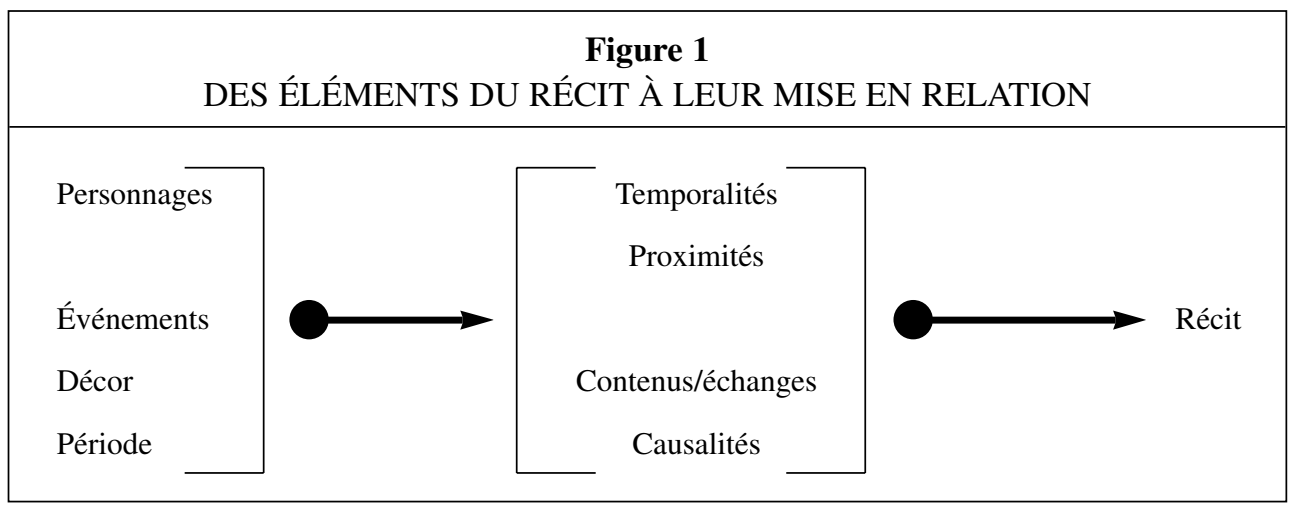


brutale et/ou chaotique (il faut brusquement accélérer ou retarder l'ouverture du site iTune en Europe). De nouveaux événements là pénètrent (la montée en puissance des mémoires flash), soit qui ont été anticipés et souhaités, soit par hasard ou parce que l'histoire en train de se faire rend leur présence indispensable. Dans cette construction, ce qui survient est en grande partie influencé par ce qui s'est passé précédemment et ainsi le récit prend forme (si il n'y a pas le succès de l'iPod, l'iShuffle ne sera sans doute pas; sans le rôle crucial du design dans le succès de l'iBook et la contre-culture d'Apple, l'iPod ne peut sans doute pas se matérialiser en objet iconique). Progressivement un ordre et une cohérence qui contraignent le déroulement futur du récit se construisent, définissant des repères pour le stratège tout autant que pour le romancier face à une histoire qu'il maîtrise plus ou moins et qui s'inscrit progressivement dans la réalité.

Mais une histoire ne se résume pas un ensemble d'éléments et à leur ordonnancement car sinon un dictionnaire ou un annuaire feraient l'affaire. Pour qu'il y ait récit, il faut également qu'il y ait une construction de sens au service d'une intention ${ }^{3}$.

\section{Construction de sens et intention au sein du récit}

Raconter une histoire, c'est comme nous l'avons vu plus haut rapporter une progression des événements au cours du temps (la séquence), ordonner les éléments les uns à côté des autres (la figure) mais aussi démasquer les contenus et leurs destinations, les échanges et les structures causales qui unis- sent les éléments constitutifs (personnagesacteurs, événements, décor, période). Cependant, parce qu'elles sont stratégiques, les narrations doivent également faire un point et pour cela il faut qu'il y ait une intention et du sens. S'intéresser à l'intention et au sens, c'est selon Adam quitter la dimension structuraliste et sémiotique du récit centrée sur son contenu immanent pour s'intéresser à sa dimension sémantique (Adam, 1984).

Ricœur, à propos de la construction de sens évoque une deuxième mimesis (Ricœur, 1966). Celle-ci consiste à prendre ensemble des facteurs hétérogènes et une succession d'actions pour en faire un tout organisé ayant un commencement, une fin et une logique. Pour Weick, cette construction de sens (sense-making) est au cœur de la stratégie (Weick, 1995). Toutefois, la spécificité de la narration, c'est qu'elle ne peut tout dire. Les macropropositions qu'elle effectue résultent autant d'un tassement de l'information (résumé) que d'une expansion (inférences) à partir du texte (Adam, 1984). Pour le stratège, construire du sens au sein d'une narration, c'est d'une part, ordonner le réel afin d'expliquer en quoi les événements et trajectoires passés préfigurent ceux à venir. C'est d'autre part, imaginer des trajectoires futures possibles et déterminer, parmi celles-ci, celles qui semblent les plus intéressantes à poursuivre. Dans le premier cas, construire du sens par déduction en sélectionnant, dans l'ensemble des éléments à disposition, ceux qui sont le plus susceptibles de rendre compte de manière compréhensible et convaincante du passé et du présent. Dans le second cas,

3. Cf. note précédente sur la relation du récit stratégique à la notion d'intrigue. 
construire du sens par induction en imaginant et sélectionnant, dans l'ensemble des éléments qui pourraient être engendrés et/ou advenir, ceux qui paraissent les plus porteurs de potentialités et de crédibilité. Pour construire et mettre en œuvre une stratégie, une organisation doit donc constamment choisir et ordonner parmi ce qu'elle peut connaître et construire à partir de cela. Dans les deux cas, proposer des enchaînements et des liens. Ceux-ci seront pour leur plus grande part prouvés et constitutifs du réel pour ce qui concerne le passé et le présent (déduction), alors qu'ils ne seront essentiellement que des hypothèses ou des projections pour ce qui concerne le futur (induction).

Ainsi Apple est dans le constat et la déduction quand il analyse la montée en puissance de la distribution en ligne sous sa forme piratée et le problème qu'elle pose aux producteurs et distributeurs. Il est par contre dans la projection et la fiction quand il envisage la possibilité de construire un modèle économique viable sur la distribution légale de cette même musique et la possibilité d'y faire adhérer les « majors ».
La construction de sens se fait également au service d'une intention. Cette intention est à la base de ce que souhaite le narrateur et de l'attente de celui qui écoute ou lit un récit (Adam, 1984). Bakhtine dans son principe dialogique (Bakhtine, 1978) et Ricœur dans son troisième mimesis (Ricœur, 1983) traitent de cette intention mais la situe du côté du lecteur qui reçoit et interprète. « La textualité se définit ainsi par l'ouverture structurale en direction du lecteur/auditeur défini comme interprétant »(Adam, 1984). Mais cette intention se situe également au centre de celui qui énonce le récit, du côté du narrateur ${ }^{4}$. La stratégie poursuit une intention, celle de guider l'action pour transformer le réel et la narration est destinée à aider en cela. Lorsque Steve Jobs se met en scène dans un Apple Expo pour raconter que sa firme va ouvrir un site de musique en ligne payant, qu'il a conclu pour cela des accords avec les «majors » et qu'il met sur le marché l'iPod, il énonce un récit stratégique dont il sait qu'il sera décrypté, analysé et jugé. Dans ce récit, il y a l'intention de communiquer, de convaincre mais aussi de changer le décor et les règles du jeu dans le secteur de la distribution de la musique.

\begin{tabular}{|l|l|l|}
\hline \multicolumn{3}{|c|}{ Tableau 1 } \\
\hline \multicolumn{2}{|c|}{ CARACTÉRISTIQUES COMPARÉES DE LA DÉDUCTION ET DE L'INDUCTION } \\
\hline & Déduction & Induction \\
\hline Processus & Sélection & Inférences \\
\hline Temporalité & À partir du passé et du présent & Orienté vers le futur \\
\hline $\begin{array}{l}\text { Nature prédominante } \\
\text { des éléments incorporés }\end{array}$ & $\begin{array}{l}\text { Essentiellement prouvés } \\
\text { et constitutifs du réel }\end{array}$ & $\begin{array}{l}\text { Essentiellement hypothétiques } \\
\text { et constitutifs d'une fiction }\end{array}$ \\
\hline
\end{tabular}

4. Adam considère la mise à l'écart de cette dernière dimension comme sans doute le principal point aveugle dans les différentes mimesis énoncées par Ricœur (Adam, 1984). 


\section{La légitimité du narrateur: position institutionnelle et « promesses d'avant la promesse »}

Si en physique, la position et la nature de l'observateur influent sur l'observation, en narration la position et la nature de l'émetteur ont la même conséquence pour le récit. C'est une des récurrences de la critique littéraire du $\mathrm{XX}^{\mathrm{e}}$ siècle que de s'intéresser à la position de l'auteur et à celle du narrateur à l'extérieur ou au sein du récit. Une histoire peut se raconter par de multiples voix $^{5 / 6}$. Les personnages peuvent échapper à leur auteur ou se rebeller contre lui ${ }^{7}$. L'auteur n'est pas forcément un démiurge. Il est contraint par l'existence des personnages qu'il cherche à faire vivre d'une part, et d'autre part par le lecteur auquel il s'adresse et dont il cherche l'adhésion ou à tout le moins la participation. Dans une narration, personnages et lecteurs ont une autonomie et une capacité à entrer ou non dans le récit conçu par l'écrivain. La légitimité de l'auteur (sa position, son expérience, ses réseaux) entre en ligne de compte. L'auteur débutant n'est pas dans la même position que celui qui bénéficie d'une expérience, de ressources et de réseaux d'influence et/ou d'action. Il en est de même dans le cas de la narration stratégique. Steve Jobs, lorsqu'il entreprend d'orienter Apple du secteur informatique vers celui de la distribution de musique en ligne, est dépositaire d'une position qui le légitime dans l'histoire qu'il propose. Il a été à l'origine d'Apple et de son renouveau. Il est également le dirigeant et principal actionnaire d'une firme et d'une expérience dans le secteur du contenu créatif puisqu'il est le directeur de Pixar, producteur de dessins animés et détenteur de succès mondiaux au box office (Toy Story I et II, Nemo). Pour cette raison, le récit stratégique qu'il propose porte au-delà de ce qui serait le cas s'il était proposé par un autre dirigeant moins bien doté en termes de pouvoir institutionnel, de ressources et de réseaux.

Mais au-delà de la position, le « track record » et notamment « la promesse d'avant la promesse » comptent également. Quand Steve Jobs énonce son récit, il effectue une double promesse. D'une part, positionner Apple sur une ligne de croissance de ses revenus et de ses profits plus forte que celle que lui offre aujourd'hui l'informatique. D'autre part, arriver à ce que ne se reproduise pas ce qui s'est produit pour Apple dans le secteur informatique où le succès initial de son format propriétaire (MacOS) est devenu progressivement sa prison tandis qu'un standard ouvert (Windows) s'imposait rapidement et offrait à ses propres promoteurs un profit durable bien plus important. Tout cela est présent à l'esprit de ceux qui évaluent la narration stratégique proposée par Apple. Ils jaugent le narrateur au regard de ses promesses antérieures, des « promesses d'avant la promesse » et de leur respect. Que voient-ils? Un dirigeant qui a su changer le monde de l'informatique comme il l'annonçait (même si d'autres en ont mieux profité) en faisant

\footnotetext{
5. Nous choisissons ici de ne pas aborder la question des positions possibles de l'auteur par rapport au narrateur. Les positions d'auteur et de narrateur sont donc considérées dans ce texte comme fusionnées.

6. Par exemple, Rashomon de Kurosawa, Le quatuor d'Alexandrie de L. Durrell, Le cercle de la Croix de I. Pears.

7. Par exemple, Six personnages en quête d'auteur de Pirandello, Si par une nuit d'été un voyageur de Calvino.
} 
de l'ordinateur personnel un objet familier au sein des foyers. Un dirigeant qui a effectivement annoncé qu'il allait remettre sur les rails une société qui perdait de l'argent et qui l'a fait en misant sur le design et la simplicité d'utilisation de ses produits. Steve Jobs est donc légitime non seulement par les ressources qu'il commande mais également par le respect de la «promesse d'avant la promesse » (Ricœur, 1983) sur lequel s'appuie la nouvelle promesse qu'il effectue au sein de sa narration (Kahane et Reitter, 2002).

Ainsi, pour être stratégique, la narration se doit de respecter un premier ensemble de conditions (dites de cohérence interne). Celles-ci concernent la sélection des éléments et leur mise en relation dans le cadre d'un agencement choisi, la création de sens au service d'une intention, la légitimité du narrateur. Mais à se limiter à la cohérence interne, on prend le risque de dériver vers des constructions qui peuvent laisser perplexes dans leurs justifications, qui sont souvent fortement teintées de relativisme et qu'il devient parfois difficile de tester de manière claire quant à leur validité ou à leurs limites. Au-delà de la manière dont des organisations construisent leur narration au regard de la cohérence interne, il nous faut donc comprendre comment d'autres formes de cohérence peuvent permettre de dépasser cet écueil du relativisme. À notre sens, ces formes de cohérence complémentaire se situent à l'extérieur du récit et contribuent à sa dimension et son impact stratégique. D'un côté, la prise en compte des audiences auxquelles s'adressent le narrateur. De l'autre, la prise en compte du réel en sus de la fiction et de l'action en complément de la narration et de l'action.

\section{II. - LA COHÉRENCE EXTERNE DE TYPE I : \\ LES CONDITIONS LIÉES AUX AUDIENCES DES RÉCITS}

Dans ce qui constitue notre second temps, nous considérons qu'un récit, s'il veut avoir une portée stratégique, ne peut se contenter de répondre à des conditions de cohérence qui soient seulement internes. À notre sens, le caractère stratégique d'une narration tient, parallèlement aux conditions internes de cohérence qu' elle doit respecter, à une capacité de communication, de conviction et de mobilisation vers des audiences. L'examen des conditions de cohérence liée à celles-ci (cohérence externe de type I) amène à s'intéresser à la rationalité narrative qui sous-tend le récit, à l'intéressement des acteurs et aux témoins de leur engagement.

\section{Rationalité narrative au sein du récit}

Pour être stratégique, le récit doit communiquer afin de convaincre (en interne et en externe) et d'influer sur le réel (au sein et à l'extérieur de l'organisation). Fisher considère qu'en matière de communication, la narration dispose d'un intérêt et d'une force spécifique (1989). Celle-ci tient à une rationalité propre (rationalité narrative) alternative à la rationalité argumentative (Weick et Browning, 1986). Fisher conçoit les êtres humains comme étant pour l'essentiel des conteurs d'histoire. Pour lui, cette dimension prévaut sur leur rationalité (ou a minima constitue une forme complémentaire à la rationalité argumentative traditionnelle).

Dans la rationalité argumentative, la prise de décision est essentiellement fondée sur la séparation des faits des conjectures, les 
réponses de type oui/non ou vrai/faux, les catégories logiques, le débat éclairé, la détection des erreurs, les démonstrations, les propositions et les délibérations. Elle présuppose que : a) le premier mode de prise de décision consiste à utiliser des structures implicatives (argument) claires et inférentielles; b) la rationalité est déterminée par la connaissance du sujet, sa capacité argumentative et son habilité à utiliser les règles logiques; c) le monde est un ensemble de puzzles logiques qui ne peuvent être résolus que par l'analyse et la mise en œuvre d'arguments raisonnés; d) la décision requiert la participation de personnes qualifiées qui soient compétentes dans l'argumentation et aient les connaissances techniques nécessaires pour juger ; e) la rationalité implique qu'il existe un système hiérarchique au sein duquel certains sont qualifiés pour juger et conduire là ou d'autres le sont pour suivre; f) le fondement philosophique sous-jacent est épistémologique et le matériel linguistique pertinent contient des propositions auto-évidentes, des démonstrations, des preuves et des expressions verbales d'une connaissance certaine ou probable (Zey-Ferrel, 1981).

Dans la rationalité narrative, la prise de décision humaine se fait selon Fisher (1989) en fonction de « bonnes raisons ». Les bonnes raisons sont les éléments qui fournissent au sujet des incitations pour « accepter et adhérer » à une position. Elles se fondent sur un raisonnement où le discours argumentatif n'est pas nécessairement lié à des structures inférentielles et implicatives claires et sans ambiguïtés, où il n'est point besoin d'être préalablement doté d'une expertise pour adhérer et participer (alors que l'expertise est nécessaire pour pouvoir argumenter avec succès). La ratio- nalité argumentative présuppose des acteurs « éclairés » aptes à débattre ensemble sur des faits et des connaissances communes ce qui nécessite que celles-ci existent et qu'elles soient partagées (ce qui est souvent difficile en raison des conflits d'interprétation) tandis que la rationalité narrative ne nécessite pas ce type de préalable. Les acteurs savent ce qu'ils savent et testent les histoires qui leur sont proposées, telles qu'elles se présentent pleines d'incertitude et d'ambiguïtés en s'identifiant à elles et en les comparant de manière analogique par rapport à leurs propres histoires. Selon Fisher, la rationalité narrative repose sur un composant formel (probabilité narrative) et un composant substantif (fidélité narrative).

Le composant formel, la probabilité narrative, s'attache aux problèmes de cohérence, aux degrés de contradictions rencontrés au sein du récit, à la continuité dans le comportement des personnages. Pour évaluer la probabilité narrative, une audience compare et contraste la narration qui lui est adressée avec les autres récits qu'elle peut connaître par ailleurs à partir de son expérience passée et présente. Elle se pose la question de savoir si des questions ou des faits pertinents n'ont pas été omis.

Le composant substantif, la fidélité narrative, s'attache à la relation du récit à la vérité. Une narration vraie est une histoire qui non seulement s'appuie sur des raisons mais surtout sur de «bonnes raisons » ancrées dans l'histoire, la biographie et la culture. Un raisonnement juste respecte la vérité et la fiabilité et sera évalué par la logique des bonnes raisons ainsi que par la logique formelle et informelle lorsque cela sera approprié. 
Une histoire doit être cohérente et crédible. Toutes les histoires ne sont donc pas possibles et en cela, selon nous, la narration à visée stratégique n'est pas relativiste. D'un côté, la probabilité narrative veut qu'une histoire pour être crédible soit cohérente. De l'autre, la fidélité narrative veut que chaque destinataire de la narration (dont le narrateur lui-même) teste les histoires proposées par rapport à un ensemble d'éléments liés à son passé, à ses connaissances et à sa manière d'interpréter le monde. Certaines histoires sont donc plus crédibles que d'autres parce qu'elles sont plus robustes en elle-même (probabilité narrative) ainsi que par rapport à ceux à qui elles s'adressent (fidélité narrative).

Au regard de la rationalité narrative, la narration tout comme une métaphore, tire son pouvoir de sa capacité à capturer des expériences complexes qui combinent le sens, la raison, l'émotion et l'imagination. La narration permet de combiner tous ces éléments ensemble et préserve leurs interactions dans un résumé compact qu'il est possible de reconstruire à partir de l'un quelconque de ses éléments (Weick et Browning, 1986). La narration offre également au sujet la possibilité de s'identifier et de se projeter au sein du récit. U. Eco, dans son livre Lector in Fabula, parle à ce sujet de l'interprétation du texte par le lecteur coopérant. Là où la rationalité argumentative repose sur la délibération, la rationalité narrative est basée sur l'identification et l'analogie (LeonardBarton, 1996). En termes d'identification et d'auto-projection, la rationalité narrative offre plus de liberté et de possibilités que n'en laisse la rationalité argumentative. Puisque la préoccupation principale n'est plus de démontrer mais de remporter l'adhésion, il devient possible d'explorer et d'utiliser la panoplie complète de la fiction et des intuitions. Ainsi, dans la rationalité narrative, la question par rapport à une stratégie ne réside plus selon nous dans une démonstration d'une véracité immanente et/ou d'un optimum. Cela est souvent impossible et nul ne peut par ailleurs affirmer avec certitude ce qui serait advenu si d'autres options avaient été prises. Elle cherche simplement à convaincre de sa plus forte plausibilité et des potentialités qu'elle offre au sujet qui s'identifie en son sein.

Lorsque Steve Jobs entreprend de positionner Apple dans le secteur de la musique, il est en mesure de fournir un certain nombre de « bonnes raisons » qui, sans se contredire, semblent suffisamment complètes. Celles-ci sonnent juste aux employés, consommateurs, partenaires, concurrents, analystes financiers et médias par rapport à ce qu'ils peuvent en connaître et à ce qui leur paraît vrai. En regard de la narration proposée, ceux-ci s'identifient dans l'histoire à venir. Ils la reconstruisent de leur propre point de vue en se glissant dans les interstices qu'elle offre pour cela et l'évalue en fonction de leurs intérêts propres.

\section{Intéressement des acteurs : la structure dialogique de la narration}

Pour être stratégique, la narration émise doit rencontrer des audiences multiples (dont le narrateur lui-même) qui le reçoivent, le réinterprètent et le relaient (éventuellement modifiée) plus ou moins fortement. Bakhtine (1978) et Todorov (1966) ont exploré le principe dialogique qui unit l'auteur et son lecteur dans une construction où les intentions, le vécu et l'imaginaire de l'un renvoie à celui de l'autre. C'est ce que Ricœur identifie comme la troisième mimesis ou « le plan de la refiguration aval du texte marque l'inter- 
section du texte et du monde de l'auditeur ou du lecteur. L'intersection, donc du monde configuré... et du monde dans lequel l'action effective se déploie et déploie sa temporalité spécifique » (Ricœur, 1983). Le lecteur a la capacité de s'identifier. Selon l'interprétation qu'en donne Adam (Adam, 1984), « le moment ou le lecteur s'approprie le monde de l'œuvre se trouve encore dans l'œuvre elle-même... En d'autres termes, l'effet produit par le texte, cette reconfiguration de l'expérience du lecteur que la lecture effectue, n'est pas vraiment extérieur au texte luimême et à sa signification ». Ricœur considère que c'est « dans l'auditeur ou dans le lecteur que s'achève le parcours de la mimesis ». Selon nous, dans la narration stratégique, la mimesis ne s'achève pas ainsi. Les audiences sont en mesure non seulement de s'identifier mais également de reconfigurer le récit proposé, d'en explorer l'impact individuel et collectif, d'en évaluer les conditions et intérêts de leur éventuelle participation. De plus, les audiences sont elles-mêmes constituées d'acteurs aptes à répondre à ces narrations et à en émettre d'autres qui viendront se confronter ou s'hybrider à la narration initiale (Czarniawska, 2002). Il y a donc en stratégie des narrations multiples et possibilité pour celles-ci d'entre-définition au sens de ce qui se produit pour les acteurs dans la théorie de l'acteur-réseau de Callon (Callon et Latour, 1981).

Callon et Latour insistent sur l'importance de l'alignement des intérêts et des stratégies des acteurs dans le succès des innovations. Cet alignement passe selon nous en partie par la capacité de ces acteurs à faire converger leurs narrations par l'échange. L'hybridation et la confrontation des narrations permettent de continuer leur enrichissement et à chaque narrateur de préciser le sens et l'intention poursuivie. "Information may generate thousands of reverberating inferences constituing a pattern (Bowers et Bradac, 1982). Through transmission, information is

Figure 2

L'ENTREDÉFINITION DES ACTEURS, DE LEUR INTÉRESSEMENT ET DE LEURS NARRATIONS ${ }^{8}$

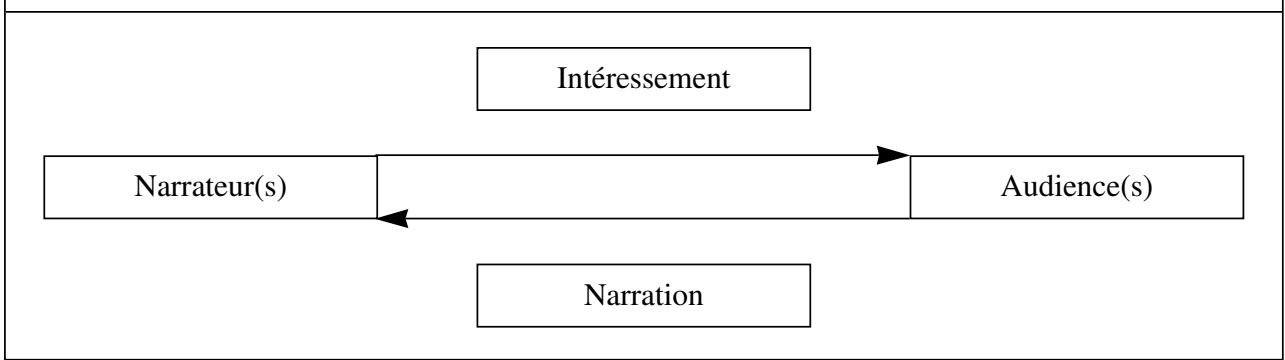

8. Vis-à-vis des narrations produites, les rôles de narrateur et d'audience sont interchangeables. Le narrateur émet une narration que l'audience reçoit. Celle-ci répond par son intéressement éventuel mais également par l'émission en retour d'une narration. L'audience devient de ce fait narrateur tandis que le narrateur initial voit son rôle transformé en celui d'audience. La boucle ainsi formée évolue pour aboutir à une entredéfinition des acteurs, de leur intéressement et de leur narration. 
qualitatively changed, made more or less meaningful (Axley, 1984)". Selon les cas, les multiples narrations évoluent dans le sens de la convergence, ou à l'instar de ce que décrit Ezrahi (1998) en sciences politiques, elles peuvent demeurer en compétition, s'affrontant et se défiant sur un champ de bataille pour capturer les cœurs et les esprits. À ce jeu, en fonction de la structure de la narration, de la légitimité du narrateur et des règles de rationalité narrative, toutes les narrations ne se valent pas. Et si l'on remonte à ce qui a été écrit précédemment quant à la cohérence interne des narrations et à la rationalité narrative, toutes les narrations ne se valent pas. Les narrations des différents émetteurs-audiences ont selon nous, compte tenu de ces différents facteurs, des poids et des impacts stratégiques plus ou moins importants.

Dans le cas d'Apple, la narration de Steve Jobs, d'une part, se superpose, à celles des producteurs de disques et des « afficionados » de la marque qui la renforcent et, d'autre part, se trouve en concurrence avec celles de Sony, de Dell, de Microsoft ou de ceux qui piratent les fichiers de musique en ligne. Chacun interprète en fonction de ses contextes et intentions propres le message de l'autre pour le transformer plus ou moins fortement. Cette interaction entre différentes parties plus ou moins puissantes, plus ou moins inventives, plus ou moins mobilisées, se produit dans le cadre d'une dynamique évolutive où les acteurs s'influencent mutuellement par la force de la cohérence interne et de la rationalité narrative de leurs récits. Parallèlement, l'intéressement réciproque des différents narrateurs/audiences aux positions et aux légitimités asymétriques vient influer sur la capacité stratégique de la narration. Mais au-delà de l'intéressement, la cohérence d'une narration stratégique est également liée à leur engagement. De cet engagement témoignent les promesses et prises de risque qui sont faites.

\section{L'engagement des narrateurs : promesses et prises de risque au sein de la narration}

Pour qu'un narrateur puisse intéresser ses audiences à ses intentions propres, il faut que ce qu'il dise l'engage d'une manière ou d'une autre (Kahane et Reitter, 2002). Un narrateur se trouve donc dans une position où pour intéresser ses audiences (et luimême) à ses projets, il lui faut s'engager et engager les autres sur ce que sera demain. Montrer comment ce qu'il souhaite réaliser est porteur de potentialités favorables aux parties qu'il cherche à impliquer et comment ce qu'il énonce l'engage directement. Un narrateur est donc porteur d'une promesse pour les autres et d'une prise de risque pour lui-même (Kahane et Reitter, 2002). Plus la promesse est attractive pour les autres, plus ceux-ci sont susceptibles de s'y intéresser et d'y engager eux-mêmes des ressources. Plus les engagements que prend un narrateur sont importants, plus sa prise de risques est forte et plus sa promesse devient crédible. Salancik (1977) a insisté sur l'importance stratégique de l'engagement et sur les irréversibilités liées à la spécificité plus ou moins forte des ressources engagées. Entre les différents émetteursaudiences qui participent à la construction et à l'évolution de la narration se met en œuvre une économie de l'engagement, ou plus précisément des engagements respectifs de chacun. Chacun est porteur d'une promesse quant à son engagement et assume une prise de risque par rapport à 
celui-ci. Là encore, promesses et prises de risque des différents acteurs ne sont pas forcément symétriques. Comme représenté dans la figure 3, nous considérons que cette économie repose sur quatre éléments : l'ampleur de la (des) promesse(s) effectuée(s), la probabilité de sa (leur) réalisation, l'importance des ressources qui lui (leur) sont consacrées, le degré de risque encouru si jamais la (les) promesses venai(en)t à ne pas se réaliser.

Lorsque Apple organise le lancement couplé du iPod (l'appareil pour écouter la musique) et de iTune (le logiciel par lequel se fait le téléchargement et la gestion du catalogue de musique sur le internet et sur son lecteur, l'iPod), il s'engage lui-même ainsi que sa firme dans la narration qu'il énonce. Sa promesse est attractive par son ampleur en termes de profits et de transformation du secteur et des pratiques. Elle a une probabilité importante de se réaliser compte tenu des accords passés avec les « majors », de la qualité et du design des produits et services proposés, de la préemp- tion opérée sur les capacités de production d'un composant crucial (mini-disque dur et mémoires flash de grande capacité). Apple a déjà consacré et va continuer de consacrer à cette promesse des ressources importantes en termes de développements et de capacité de production, de marketing et de vente. Enfin, le choix effectué conditionne une part importante de la capacité de la firme à rester un acteur majeur à l'heure où les parts relatives de ses micro-ordinateurs, bien que profitables, diminuent. Si le choix s'avère erroné, c'est à terme probablement l'avenir de la firme qui sera menacé. En regard, un consommateur qui achète un iPod effectue une promesse de consommation des services de iTune et de généralisation du standard mais assume une prise de risque si le standard vient à décliner, diminuant la valeur de son achat.

Les narrations pour avoir un caractère stratégique doivent donc non seulement répondre aux conditions de cohérence de type interne mais également à celles qui concernent les audiences auxquelles elles

Figure 3

UNE ÉCONOMIE DES ENGAGEMENTS RESPECTIFS

\begin{tabular}{|c|c|c|}
\hline $\begin{array}{c}\text { Ampleur } \\
\text { de la (des) } \\
\text { promesse(s) }\end{array}$ & & $\begin{array}{c}\text { Probabilité de réalisation } \\
\text { de la (des) } \\
\text { promesse(s) }\end{array}$ \\
\hline $\begin{array}{c}\text { Ressources } \\
\text { consacrées }\end{array}$ & & \\
\hline
\end{tabular}


se relient (cohérence externe de type I). Pour autant, en rester à cela, c'est de manière implicite inférer que les narrations évoluent dans un univers qui demeurerait relativiste. La force de conviction se suffirait à elle-même sans que le réel et les lois de la nature, de la science et des rapports entre les hommes ou les organisations ne soient déterminants. Bien entendu, il n'en est rien. En effet, la narration stratégique ne peut se concevoir sans que la fiction contenue dans la narration ne fasse référence constamment au réel et sans que la narration trouve sa contrepartie sous forme d'action. Au-delà de ce qui concerne la relation aux audiences, cette nécessité d'un couplage de la fiction au réel, de la narration à l'action définissent des conditions de cohérence externe de type II.

\section{III. - LA COHÉRENCE EXTERNE DE TYPE II : LES CONDITIONS DE COHÉRENCE LIÉES AU RÉEL ET À L'ACTION}

Dans ce troisième temps, nous montrons que le passage de la narration à la stratégie nécessite une double extension. D'une part, si la stratégie nécessite un ancrage dans le registre du réel, la narration offre, par son possible recours à la fiction, la capacité d'un décrochage momentané du réel afin de mieux y revenir par la suite. D'autre part, si la narration est liée au registre du verbe, la stratégie implique une mise en œuvre par l'action qui est indispensable pour démontrer la validité et la performance que la narration propose. Par la fiction au sein de la narration, l'organisation peut proposer des futurs possibles et des voies pour y parvenir. Par l'action, elle démontre sa capacité à transformer en réalité ces fictions sur des futurs possibles.

Pour témoigner de ces croisements, nous procédons en trois temps. Tout d'abord, nous nous intéressons aux complémentarités et aller-retour entre réalité et fiction. Puis nous examinons le récit en tant que générateur d'action. Enfin, nous traitons de l'action, dans sa capacité à alimenter la narration.

\section{Réalité et fiction : d'un réel présent à un réel futur en passant par la fiction}

Pour Greimas (1966), la narrativité apparaît comme « l'irruption du discontinu dans la

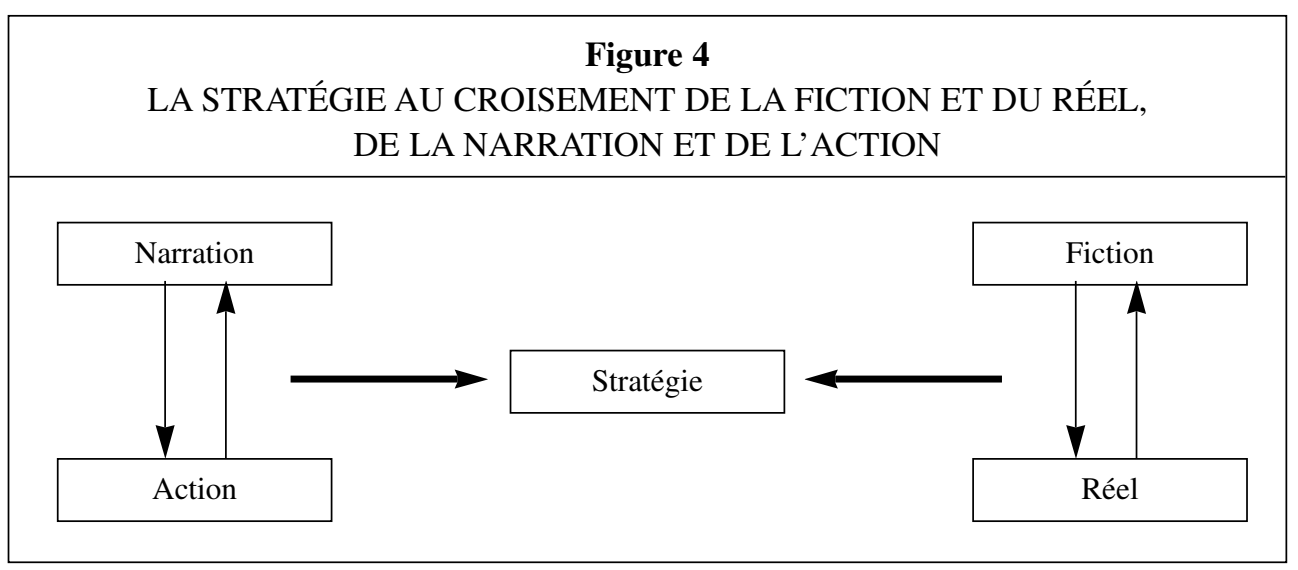


performance discursive d'une vie, d'une histoire, d'un individu, d'une culture », irruption qui introduit des états entre lesquels apparaissent des transformations. Pour une organisation, se projeter dans le futur est un moyen d'introduire de la discontinuité tout comme une discontinuité conduit à se projeter dans le futur. Pour que cette projection s'opère, il faut pouvoir se détacher du réel et pour cela procéder par la fiction. Imaginer ce que serait un futur autre et les voies pour l'atteindre. L'introduction de la fiction au sein du récit permet la prise en compte du hasard et évite les écueils de la démonstration. Elle permet de jouer avec les différentes possibilités de ce qui pourrait être. Au sein de la narration, la part fictionnelle peut être plus ou moins forte, selon l'horizon plus ou moins lointain dans laquelle la narration projette, selon le degré de discontinuité poursuivi, selon l'état des connaissances disponibles et à acquérir pour rétablir la continuité.

Toutes les fictions ne sont pas possibles par rapport au réel présent et à venir. La construction stratégique qu'opère Apple ne se fait pas dans un absolu mais dans un univers limité de possibles plus ou moins plausibles qui s'ils ne les déterminent pas, du moins en partie, contraignent les choix de la firme. Il existe en effet des lois de la nature et de la science inviolables ou à tout le moins difficilement contournables ainsi que des régularités de l'action humaine et organisationnelle. Il y a cependant un abîme entre ces deux types de composantes. Une loi ne pourra jamais tout prévoir (la corde du pendu peut toujours se rompre) mais n'en demeure pas moins vraie du fait de son abstraction. Elle pose ses conditions et prédit sous couvert de celles-ci, « toutes choses étant égales par ailleurs ». À l'in- verse, dans le monde de l'action organisationnelle et humaine il y a des particularités dans le temps et dans l'espace et il peut y avoir des exceptions. Avoir constaté une fois un enchaînement causal, c'est certes s'attendre à le voir se répéter mais cela ne va pas jusqu'à la certitude. Cette présence de l'événementiel modifie radicalement la nature des prédictions sur lesquelles peut s'appuyer l'organisation dans ses projections. Ce ne sont plus des lois universelles qui permettent de prévoir mais des causes particulières qui expliquent. L'homme est libre, le hasard existe, les événements ont des causes dont l'effet demeure douteux, l'avenir est incertain et le devenir contingent. Il y a donc toujours une marge de flou et d'aléatoire. Ceci explique pourquoi l'organisation ne sait jamais totalement de quoi demain sera fait. Trois raisons sont selon nous à la source de cette indétermination: La première est due à l'existence d'événements qui peuvent perturber le cours de l'action humaine et organisationnelle. $\mathrm{La}$ seconde tient à l'innovation scientifique et technologique dont la raison d'être et le but avoué sont d'introduire de nouveaux éléments au sein de ce qui est connu afin de le modifier. La troisième est liée à la variabilité dans l'interprétation des causes et résultats liés à ces événements, à l'action humaine et organisationnelle ainsi qu'à l'innovation scientifique et technique. Cette indétermination engendre une flexibilité qui offre de multiples possibilités à la fiction. Or, selon nous, l'un des intérêts et des légitimations majeurs de la narration en ce qui concerne la stratégie tient à cette capacité de mobilisation et d'intégration de la fiction au sein du récit.

Dans le cas d'Apple, ce sont l'impunité et la légitimation croissante du piratage au 
sein de la société ainsi que les inquiétudes des « majors » par rapport à cette évolution qui rendent attractive le modèle initialement fictionnel de distribution légale proposé par Apple pour la distribution de musique en ligne. C'est du côté humain et organisationnel le développement d'une contre-culture fondée sur l'immédiateté, la mobilité et la gratuité qui change les conditions et les règles du jeu social. Ce sont du côté de l'évolution technique, l'internet à haut débit, le développement du « peer to peer », l'apparition de mini-disques durs de grande capacité et la montée en puissance des mémoires flash qui changent les conditions et règles du jeu technique. Combinées, ces évolutions permettent à Apple d'intéresser les « majors » à un modèle alternatif, légal et contrôlable de distribution et de se créer un marché sur lequel exister et prospérer par l'offre de ses produits et services. La société et la technique évoluent et avec cela s'ouvrent de nouvelles possibilités pour la fiction au sein de la narration.

Certaines fictions sont cependant plus risquées que d'autre. En fonction de la part d'ambiguité et d'incertitude qu'elles sont prêtes à accepter, les organisations élaborent des narrations qui se situent entre deux extrêmes: soit elles limitent la prise de risque vis-à-vis de l'encore inconnu et organisent la narration dans un contexte ou ne sont envisagés que les aspects choisis et retenus pour certains. Soit, elles acceptent un risque plus élevé et produisent une narration qui prend en compte des aspects plus larges et encore inconnus qui ont des probabilités variables de se réaliser. Le choix d'une attitude ou d'une autre est révélateur de l'identité d'une firme. La part de risque qu'une firme comme Apple est prête à assu- mer est sans doute plus importante que ne l'est celle de Dell. Le risque tout comme la contre-culture sont dans les gènes et les récits d'Apple tandis que Dell de son côté, produit des narrations stratégiques qui ont un rapport différent au risque.

Enfin, lorsque le risque est élevé, maintenir l'organisation dans le seul registre de la fiction est une tâche ardue. La narration peut dans ce cas aider à maintenir la fiction en vie en annonçant les étapes de sa réalisation. En proposant un enchaînement intégré de mobilisations, de dispositifs, de tests qui permettent de relier le présent au futur en train de se faire, elle peut contribuer à réduire le risque encouru. Chacune de ces étapes est l'occasion d'un test de la fiction par le réel à partir duquel se confirme ou s'infirme les objectifs poursuivis et la trajectoire empruntée. Ainsi, tout comme l'innovation dans le modèle de Callon et Latour (Callon et Latour, 1981), la narration avance en spirale, oscillant entre des phases de proposition narrative par la fiction et des phases de validation narrative par le réel. De la capacité de la firme à détecter les évolutions de son contexte et de sa flexibilité organisationnelle (Kahane, 2003) dépendra sa potentialité à adapter sa narration et son action. Dans le cas d'Apple, la firme fait des hypothèses sur le développement du marché légal de la distribution en ligne et sur l'attractivité de son offre. Celles-ci seront ensuite validées ou non par le marché. À plusieurs reprises lors des fêtes de fin d'année, les projections effectuées en termes de vente vont se trouver dépassées et conduire à un réexamen du récit proposé. Ce qui avait été imaginé ne s'est pas produit ou plus exactement ce qui s'est produit a été au-delà de ce qui avait été imaginé. De même, la fiction initiale d'une 
firme où l'activité liée à la musique équilibrerait ou l'emporterait sur l'activité informatique devient réalité et confirme Apple dans sa volonté de poursuivre et de relancer sa narration dans un sens inchangée au moyen d'une fiction similaire à celle qui a été poursuivie jusque-là.

\section{De la narration à l'action : le récit pour alimenter l'action}

Un récit ne trouve donc son sens qu'à accomplir un certain effet sur celui (ou ceux) auquel (auxquels) il est destiné (Adam, 1984). Le récit stratégique ne trouve selon nous son sens qu'à orienter l'action sur le réel. Ricœur (Ricœur, 1966) examine la question de la relation de la narration à l'action. D'un côté, dans la suite d'un de ses ouvrages précédents (Ricœur, 1966) et à l'instar de l'historien Paul Veyne, il questionne la relation du récit aux faits et à l'action du passé. De l'autre, il met l'accent sur les rapports qui interviennent entre une réflexion sur le discours et le récit et une interrogation sur l'idéologie et l'action humaine (passée, présente et future) au sein de la Cité. Ricœur considère que « les textes - principalement littéraires - sont des ensembles de signes qui ont peu ou prou rompu leurs amarres » avec les choses. À notre sens, la dimension stratégique d'une narration est directement liée à sa capacité de lien et d'impact avec, selon la formule de Ricœur, « les choses qu'ils sont censés désigner ». Ricœur traite également de ce lien et considère que « parmi les choses dites dans les textes, il y a des hommes agissant et souffrants; de plus, les discours sont euxmêmes des actions; c'est pourquoi le lien mimétique entre l'acte de dire (et de lire) et l'agir effectif n'est jamais tout à fait rompu ». Ricœur précise « $\mathrm{Au}$ point de départ, le texte et sa structuration interne, avec toutefois en sourdine son pouvoir de refiguration externe; au point d'arrivée, une esquisse du concept de raison pratique et l'irruption de l'agir dans le temps présent sous la figure de l'initiative ». Il y a donc d'un côté le « speech act », le texte qui fait action par son existence même (Brown, 1985). De l'autre, il y a le texte qui conduit à l'action par la capacité des êtres humains et des organisations à le relayer et à le mettre en œuvre (Wilkins, 1984). Wilkins considère en effet que la principale qualité de la narration est de diffuser une vision abstraite de la stratégie en des termes qui la rendent accessible, concrète et susceptible de guider l'action quotidienne. Giroux considère également que l'un des enjeux de la stratégie est dans la mobilisation des «énergies internes et des ressources externes » (p. 23). La question du lien de la narration à l'action est donc bien présente à la fois chez Ricœur et en stratégie, le rôle de cette dernière étant considéré comme celui de mobiliser les énergies et de guider l'action.

De La Ville et Mounoud (2003) analysent les relations entre narration, stratégie et pratique, cette dernière étant considérée au sens de l'action et de la microstratégie en référence aux travaux de Whittington (1993). D'un côté, elles conceptualisent la fabrication de la stratégie en tant que processus « of telling, recounting, communicating ». De l'autre, elles font référence aux catégorisations de la stratégie de Whittington (1993) en différenciant quatre approches (classique ou de planification, évolutive ou guidée par l'efficience, procédurale ou artisanale, systémique ou inscrite dans le social). Au sein de celles-ci, elles opposent les deux premières qualifiées de délibérées aux deux suivantes qualifiées 
d'émergentes. Au regard de la narration, les deux premières renvoient à une narration du stratège imposée à l'organisation tandis que les deux autres mettent en jeu une narration négociée avec celles qui existent par ailleurs au sein de l'organisation. Dans tous les cas est ainsi abordée la capacité de la narration à alimenter les pratiques et donc l'action.

Les manuels de stratégie distinguent habituellement entre formulation et mise en œuvre de la stratégie tout en insistant sur l'importance de leur couplage. Est-ce pour eux une manière autre de nous dire que narration et action sont comme Janus ou une pièce de monnaie où une face ne va pas sans l'autre. Sauf à perdre l'essentiel de sa dimension et capacité stratégique, la narration ne pourrait s'envisager hors de ce couplage à l'action. L'absence, la perte ou la faible qualité de celui-ci entraîne l'affaiblissement de l'impact stratégique et donc de la crédibilité de la narration. Ainsi, la narration stratégique est une narration pour l'action et ceci constitue la première dimension qui en fait une nar-action (Kahane, 2003). Ce couplage constitue selon nous une condition forte de la cohérence et de la capacité stratégique de la narration.

La narration d'Apple se traduit en une allocation de ressources qui la renforce. Lorsque Apple construit et énonce son récit stratégique vers ses audiences autour de son entrée dans le secteur audio, il annonce les étapes de son développement qu'il compte mettre en œuvre: l'ouverture successive de sites en différentes régions du monde, les produits à venir. Ce récit agit comme un ensemble de repères au sein d'Apple et pour les différents acteurs (développeurs, partenaires, concurrents, analystes, consommateurs) qui en tirent des conséquences et organisent leur action en fonction. L'action d'une organisation vient confirmer ou infirmer la narration proposée initialement. Si elle n'est pas telle que la narration l'envisageait, l'écart doit être justifié.

\section{De l'action à la narration : l'action pour alimenter la narration}

L'un des essais de Ricœur (1983) «Le modèle du texte: l'action sensée considérée comme un texte. » traite directement de l'action en tant que constructeur de sens au même titre que la narration. L'action est donc porteuse de sens. Dans cette perspective et en ce qui concerne la stratégie, sa construction requiert la même attention que la narration et elle impactera tout autant, si ce n'est plus, que cette dernière. De même, si pour Giroux la narration est constructiviste et constructionniste, l'action l'est tout autant. L'action, tout comme la narration, est un processus par lequel les acteurs construisent individuellement le monde dans lequel ils seront (constructivisme) ainsi que celui par lequel ils le négocient collectivement (constructionnisme). Notamment, les travaux de Weick (1995) sur l'enactement témoignent de cette capacité des organisations à créer le monde par leur action. L'action n'est donc pas seulement porteuse de sens, elle est également en mesure de transformer le réel. La capacité stratégique d'une organisation ne repose pas simplement sur sa capacité à dire le monde mais également sur celle qu'elle a d'agir sur celui-ci. La stratégie tient non seulement aux ressources dont dispose l'organisation mais également à sa capacité à les mettre en œuvre et à la manière de le faire. Il en résulte donc selon nous une série d'allers-retours entre ce que l'organisation raconte et ce qu'elle fait. D'un côté, selon 
Giroux, la narration est janusienne: « tournée vers le passé, elle rapporte avec nostalgie les succès anciens; orientée vers le futur, elle appelle à une grande aventure » (Giroux et Marroquin, 2006, p. 23). La narration reconstruit donc la mémoire au sein de l'organisation et projette l'esprit dans le futur pour préparer l'action. De l'autre, l'action selon nous l'est tout autant: tournée vers le passé, elle produit des faits qui confirment ou invalident la mémoire passée; orientée vers le futur, elle construit et réalise une grande aventure. L'action produit donc de la réalisation qui vient enrichir la mémoire et rapproche ou éloigne du futur envisagé par la narration. Narration et action se tricotent et s'entretiennent l'une l'autre, alimentant ensemble la formation de la stratégie et sa réalisation.

À chaque accord avec un nouveau « major» de la production musical ou avec un constructeur (HP), ouverture d'un site Itune de musique en ligne dans une nouvelle zone géographique, préemption sur un composant-clé (mini-disque et mémoires flash de grande capacité), introduction d'un nouveau produit, Apple construit et transforme le monde, alimentant ainsi sa propre narration. L'ouverture de l'iPod sur les PC, l'accord avec HP par lequel cette firme produit et commercialise des iPod me-too, l'accord avec Motorola pour introduire Itune dans les téléphones mobiles, les multiples générations et déclinaisons en nouveaux produits, autant de pierres qui alimentent la narration sur la généralisation d'un standard de fait et sur la capacité future d'Apple à en tirer un profit durable. Ce couplage de l'action vers la narration constitue selon nous la deuxième dimension de la nar-action (Kahane, 2003). La nar-action n'est pas seulement une narra- tion pour l'action (où la narration guide l'action) mais également une narration par l'action (où l'action alimente la narration). Lorsque la narration peine à intégrer l'action réalisée, elle perd de sa capacité et de son impact stratégique.

Considérés ensemble, les allers-retours entre fiction et réalité, l'influence de la narration sur l'action et l'alimentation de la narration par l'action, constituent le troisième type de conditions de cohérence par lesquelles la narration témoigne de sa portée et de son impact stratégique.

\section{CONCLUSION}

On en arrive donc aux constats suivants: face à des données pléthoriques et pour partie en cours de construction, la narration permet d'ordonner les éléments dans le temps et l'espace et de construire des causalités et du sens au service d'une intention. Cependant, le narrateur tout autant que les audiences peinent à pratiquer la déduction et l'induction face aux éléments à disposition. Il est souvent difficile de savoir et d'affirmer ce qui se (re)produira et à quelles conditions. La légitimité et l'expérience du narrateur entrent en ligne de compte car elles l'aident dans la construction de la narration.

Par ailleurs, pour agir sur le réel, le narrateur doit se mobiliser et mobiliser d'autres. Pour cela, il doit communiquer, convaincre et interagir avec des audiences qui sont également productrices de narrations et qui sont susceptibles d'influer sur sa narration. Dans cette interaction, il s'appuie sur la rationalité narrative, rentre dans un processus d'intéressement et d'entre-définition avec ces audiences, chacune des parties témoignant des engagements pris par les promesses et prises de risque effectuées. 
Pour se projeter, l'organisation recourt à la fiction afin de se détacher temporairement (et non pas définitivement ce qui serait de l'utopie) de l'immédiat du réel. Le réel, c'est le général du fait de l'existence des régularités humaines et organisationnelles (ce qui n'est pas coïncidence fortuite a vocation à se reproduire) et des lois de la nature et de la science (la prédictibilité liée aux lois de la nature et au caractère cumulatif des sciences). La fiction, c'est la capacité de transgression car les régularités humaines ne sont pas absolues et parce que de nouveaux éléments encore inconnus sont susceptibles de modifier ces lois par la suite. La fiction est donc bornée sans être pour autant totalement déterminée. À partir du réel et de la fiction sur lesquelles elle se construit, la narration permet de réinterpréter la mémoire et d'influer sur l'action. De son côté, l'action opère une modification du réel qui valide ou infirme la narration, changeant les conditions d'existence de la mémoire et celles d'élaboration de la narration.

Ainsi que nous l'avons exposé, il en ressort un ensemble de conditions de cohérence pour que la narration ait une capacité stratégique quant à l'organisation et à son environnement. Ces conditions de cohérence sont internes (liées aux conditions de structure pour qu'un récit existe, à ses conditions pour qu'il soit véhicule de sens et d'intention, à la légitimité du narrateur vis-à-vis de ce récit). Elles sont également externes de type I par rapport aux audiences à qui s'adresse ce récit (respect de la rationalité narrative, intéressement des audiences, promesses et prises de risque respectives du narrateur et des audiences les uns envers les autres). Enfin, au-delà de ce qui a trait aux audiences, elles sont également externes de type II, en ce qui concerne l'interaction de l'organisation avec son environnement (conditions de cohérence de la fiction par rapport au réel, capacité de passage de la

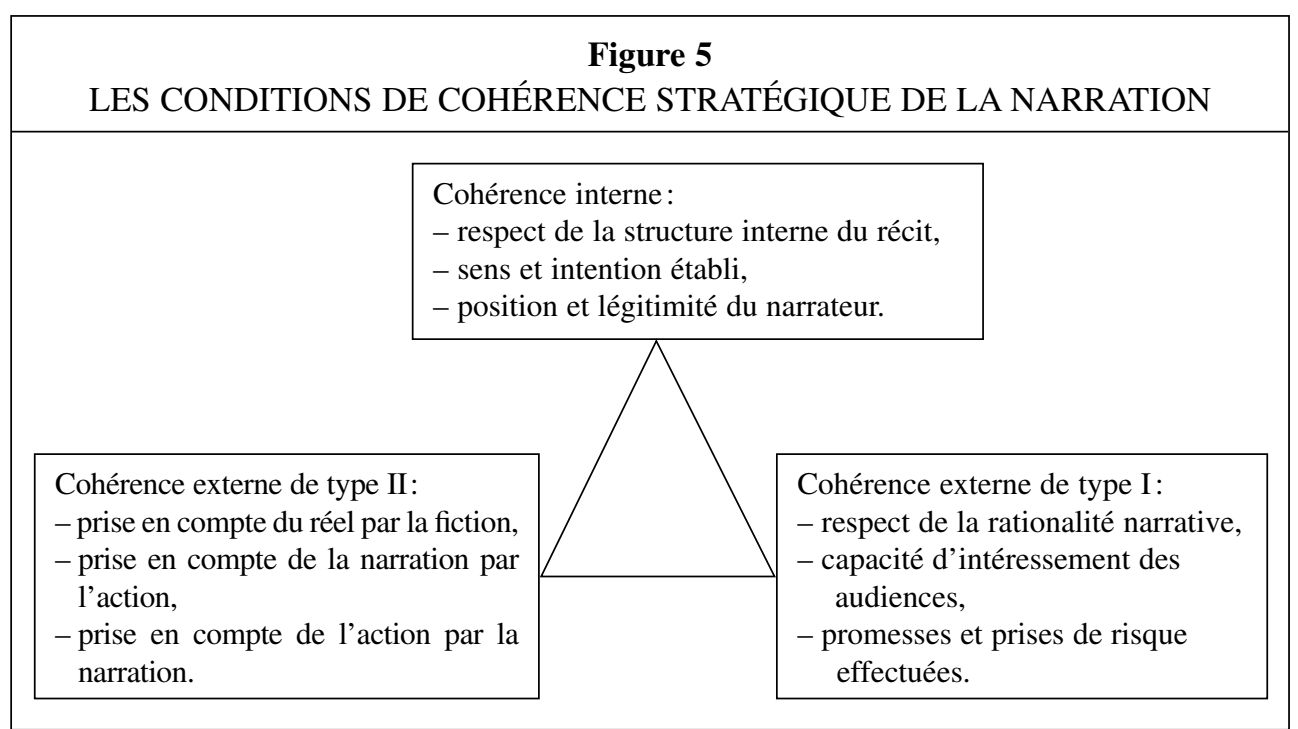


narration à l'action, capacité de l'action à alimenter la narration).

Ensemble, ces conditions font qu'une narration sera ou non nar-action, qu'elle aura ou non une capacité et un impact stratégique. Trois points méritent à notre sens d'être soulignés quant à la nar-action.

Tout d'abord, les narrations sont produites non par un narrateur unique mais collectivement en relation avec des audiences qui ont capacité elles-mêmes à être narratrices et productrices d'action. On retrouve dans la littérature et le cinéma des formes de récit/narration construites de manière similaire à partir de l'expression de plusieurs voix (cf. le film Rashomon de Kurosawa, le The Alexandria Quartet de Lawrence Durrell, la Lettre de Sibérie de Chris Marker) qui viennent chacune fournir un éclairage complémentaire à l'histoire. Ici cependant, cette narration est produite par les acteurs eux-mêmes et non par un auteur extérieur qui se projette dans ses personnages. Nous sommes donc dans un roman particulier où les personnages eux-mêmes (et non l'auteur) génèrent l'action et définissent leur place et rôles respectifs par rapport à celleci. Ceci constitue un premier point qui à notre sens contribue à faire de la narration une nar-action. Narrateurs et audiences se confondent et sont tous des narrateursacteurs qui produisent des nar-actes.

Ensuite, la crédibilité de la narration est conditionnée par sa cohérence interne (probabilité narrative) et externe (fidélité narrative) mais également par la légitimité et la capacité d'action de ceux qui y sont intéressés et y participent (leur trajectoire passée, les ressources et compétences qu'ils mobilisent, ce qu'ils accomplissent, etc.). La crédibilité de la narration stratégique se construit continuellement par les interpréta- tions croisées que de multiples acteurs et audiences portent sur les projections et les actions envisagées ou accomplies (par eux ou par d'autres) ainsi que sur leurs conséquences. Les nar-actes produits sont asymétriques par leur construction-même et parce que sont les acteurs. Ils peuvent converger ou diverger, renforçant ou épuisant les narrations initiales. Ceci constitue à notre sens le deuxième point qui enrichit la narration pour en faire une nar-action. Les naracteurs occupent des positions de légitimités asymétriques et leurs nar-actes le sont également en termes de pouvoir d'influence et de réalisation.

Enfin, la narration se construit à partir du registre de la fiction et de celui du réel, sa capacité stratégique étant fonction de l'intégration et du tricotage réalisés entre ces deux composantes. D'un côté, la narration part du réel et se sert de la fiction pour projeter l'organisation dans le futur et guider l'action. De l'autre, l'action réalise la transformation du réel et permet à la narration et à la part de fiction qu'elle contient de rejoindre celui-ci. Ceci constitue à notre sens le troisième point qui enrichit une narration pour en faire une nar-action. La narration agit comme un mécanisme qui permet, par la fiction, de suspendre la relation au réel présent et d'imaginer un réel futur (et les voies pour l'atteindre) tandis que l'action permet d'accomplir la trajectoire qui organise le retour vers ce réel futur. Plusieurs éléments peuvent dès lors entrâ̂ner la remise en cause de la capacité et de l'impact stratégique d'une narration, lui dénier son caractère de narraction et son aspect performatif. La narration peut tout d'abord être victime d'une faiblesse ou absence de cohérence interne par non respect des conditions énoncées dans ce texte. 
Il peut en être de même en ce qui concerne les conditions de relation et d'interaction avec les audiences (cohérence externe de type I). La faiblesse ou la déficience peut enfin se situer dans les conditions de cohérence de type II, dans la relation qui relie la fiction au réel ou dans le couplage nécessaire entre la narration et l'action. Selon nous, le passage de la narration à la naraction conduit à réinterpréter l'adage « Dire ce que l'on fait, faire ce que l'on dit » pour le réinterpréter afin de le renforcer pour ce qui concerne la stratégie en "Raconte ce que tu fais et va faire, fait ce que tu as raconté ». Si le nar-acteur potentiel ne raconte pas, il n'y a pas de narration mais il peut y avoir stratégie, y compris une stratégie performative (fondée sur le secret et la surprise). Si le nar-acteur potentiel ne fait pas, il n'y a pas d'action et donc pas de stratégie, seulement un récit. Si les deux sont couplés c'est-à-dire si l'adage dans sa nouvelle mouture est respecté, le nar-acteur potentiel est bien dans la nar-action et accroît la capacité performative de la narration. Si les deux sont découplés, le naracteur potentiel est hors de la nar-action et diminue la capacité performative de la narration. Les organisations agissent par leurs récits et elles racontent par leurs actes. Les audiences et le réel sont les ancrages de la nar-action, la fiction son moteur.

\section{Bibliographie}

Adam J.-M., Le récit, coll. «Que sais-je? », Presse universitaires de France, Paris, 1984, Axley S., "Managerial and organizational communication in terms of the conduit metaphor", Academy of Management Review, vol. 9, 1984, p. 428-437.

Bakhtine M., Esthétique et théorie du roman, Gallimard, Paris, 1978,

Bowers J., Bradac J., "Issues in Communication Theory: A metatheorical analysis", Communication Yearbook, Burgnon (Ed.), vol. 5, Sage, Beverly Hills, CA, 1982, p. 1-27.

Brown A., "That Remind Me of a Story: Speech Action in Organizational Socialization", The Western Journal of Speech Communication, vol. 49, 1985, p. 27-42.

Callon M. et Latour B., "Unscrewing the big Leviathan: How actors macro-structure reality and how sociologists help them to do so", Advances in social theory and methodology: Toward an integration of micro-and macro-sociologies, Knorr-Cetina K. et Cicourel A. (Eds), Routledge \& Kegan P., Boston, 1981, p. 277-303.

Czarniaswska B., Narrating the Organization, The University Press, Chicago, 1997.

De La Ville V., Mounoud E., "Between Discourse and Narration: How can strategy be a practice", Narratives We Organize By, Czarniawska B. et Gagliardi P. (Eds), John Benjamins, Amsterdam, 2003, p. 95-113.

Ezrahi Y., Rubber bullets: Power and conscience in Modern Israel, University of California Press, CA, 1998.

Fisher W. R., Clarifying the narrative paradigm, Communication Monographs, vol. 56, 1989, p. 55-58. 
Giroux N., Marroquin L., «L'approche narrative des organisations », Revue française de gestion, $\mathrm{n}^{\circ} 159$, novembre-décembre 2005, p. 15-42.

Greimas A. J., Sémantique structurale, Larousse, Paris, 1966.

Kahane B., «Comment construire le futur sans le prédire? Le modèle du système immunitaire», Cadix et Pointet (Eds), Le management à l'épreuve des changements technologiques, Organisations, Paris, 2003.

Kahane B., Reitter R., "Narractive identity: Navigating between 'Reality' and 'Fiction'”, Corporate and Organization Culture, Moingeon \& Soenen (Eds), London, Routledge Publications, 2002.

Leonard-Barton D., Wellsprings of Knowledge, Harvard Business School Press, Boston, 1996.

Propp V., Morphologie du conte, coll. « Points », Le Seuil, Paris, 1970.

Ricœur P., Du texte à l'action, Esprit/Le Seuil, Paris, 1966.

Ricœur P., Temps et récit, 3 tomes, Le Seuil, Paris, 1983-1985.

Salancik G., "Commitment and the control of organizational behavior and belief", New directions in organizational behaviour, Staw \& Salancik G. (Eds), Chicago, St Clair, 1977, p. 1-54.

Todorov T., Les catégories du récit littéraire, Communications n 8, Paris, 1966.

Weick K., Sensemaking in Organization, Sage, Thousand Oaks, 1995.

Weick K., Browning L., Argument and Narration in Organizational Communication, Yearly Review of Management of the Journal of Management, Hunt \& Blair (Eds), vol. 12, $\mathrm{n}^{\circ} 2$, 1986, p. 243-259.

Whittington R., What is Strategy and does it matter?, Routledge, London, 1993.

Wilkins A. L., "The Creation of Company Cultures: The Role of Stories an Human Resource Systems", Human Resource Management, vol. 23, n 1, 1984, p. 41-60.

Zey-Ferrel M., "Criticisms of the dominant perspective on organizations", Sociological Quaterly, 22, 1981, p. 181-205. 